\title{
SOURCE SEPARATION BASED ON COUPLED SINGLE DOA ESTIMATION PROCESSORS*
}

\author{
Montse Nájar, Miguel A. Lagunas, Ana I. Pérez-Neira \\ ETSI. de Telecomunicación, TSC Department \\ Apdo. 30002, 08080 BARCELONA, SPAIN
}

\begin{abstract}
The separation of sources from an array of sensors is addressed in this paper. Based on the Kolmogorov's theorem, an adaptive algorithm is proposed to separate simultaneously all the unknown impinging sources on an aperture of sensors. This method solves the problem of simultaneous DOA (angle of arrival) estimation and source beamforming in narrowband array processing. The Kolmogorov's theorem is implemented by a network composed of two different stages: the inhibition and the optimization stages.
\end{abstract}

\section{INTRODUCTION}

This paper deals with the so-called 'source separation' problem. Because of its numerous possible applications, in particular in the framework of antenna array processing, the attention paid to this subject has recently increased.

Following the pioneer task in the source separation problems by Herault and Jutten [1] and [2], among others, a coupled EKF scheme was developed, based on inhibitory cells and Extended Kalman Filters. The scheme uses high order learning, in order to obtain independent outputs [3]. The resulting scheme was applied to separate co-channel single tone interference with severe doppler. The reported results were in high competition with any reported scheme on high resolution spectral estimation or time-frequency analysis. The first attempt to emulate the excellent results, obtained for the time varying multifrequency tracking, in the DOA estimation problem has been reported in [4] as a preliminary work on this issue.

This paper will focus on the DOA estimation problem. Quite often the situation may be described as several sources in different directions, radiating at the same time, which impinge on an aperture of sensors. The signal received by each sensor is usually disturbed by noise from the amplifiers of the sensors. Then, the input of the processor will be a set of narrowband array snapshots. It is possible to find out the direction of arrival of the impinging sources, by means of any of the well-known array processing techniques; for instance: Music, Esprit, etc. In this paper, a new system based on the Kolmogorov's theorem, is presented.

First of all we will outline the Kolmogorov's theorem: next, an approach relying on this theorem is proposed to solve the problem of simultaneous DOA estimation and source beamforming in narrowband array processing: finally, some simulations results will be shown.

\section{KOLMOGOROV'S THEOREM}

In 1957 Kolmogorov published [5] an interesting theorem concerning the representation of arbitrary continuous functions, named the Kolmogorov's Mapping Neural Network Existence Theorem:

Given any continuous function $f(x)=y$, where $x$ and $y$ are vectors with $n$ and $m$ components respectively, the function can be implemented by a two-stage network. The first stage produces $2 n+1$ outputs and the second stage provides the desired $m$ components of vector $y$.

The first stage is formed by $2 n+1$ processors, each of them implementing the following transfer function:

$$
z_{k}=\sum_{j=1}^{n} \lambda^{k} \psi\left(x_{j}+k \varepsilon\right)+k
$$

where the real constant $\lambda$ and the continuous real monotonically increasing function $\psi$ are independent of $\mathbf{f}$ (although they do depend on $n$ ). The constant $\varepsilon$ is an arbitrary chosen positive value.

${ }^{*}$ This work was supported by the National Research Plan of Spain, CICYT, Grant number TIC92-0800-C05-05. 
The second stage contains $\mathrm{m}$ processing elements with the following transfer function:

$$
y_{i}=\sum_{k=1}^{2 n+1} g_{i}\left(z_{k}\right)
$$

where the functions $g_{i}$ are real and continuous (and dependent on $f$ and $\varepsilon$ ).

This description has been obtained from HechtNielsen [6], another important reference of this theorem is the work by Lorentz [7].

Unfortunately, no especific examples of implementation of this theorem are known. Kolmogorov's theorem is strictly an existence theorem. It tell us that such a two-stage mapping network must exist, but it does not tell us how to find it.

The objective assigned to the first stage of our approach to the Kolmogorov's theorem is to turn the multivariate problem into a single variable one. In order to describe clearly the resultant framework we will focus on the problem of two impinging sources; nevertheless, the obtained architecture can be easily generalized. Then, assuming that only two sources are present in the array scenario, a two-branch system is needed. Each of these branches has to estimate the wanted parameters of one of the sources. Thus, the first stage of the processor will convert the problem into two problems of single source DOA estimation, where both DOAs $\left(\Theta_{1}, \Theta_{2}\right)$ will be determined by the second stage, in a easier context than in the initial one. A feedback between the two stages allows the adaptive learning needed for the convergence of the method. A more detailed description of these twostage network will be provided in [8]. in figure 1:

This system can be represented by the architecture

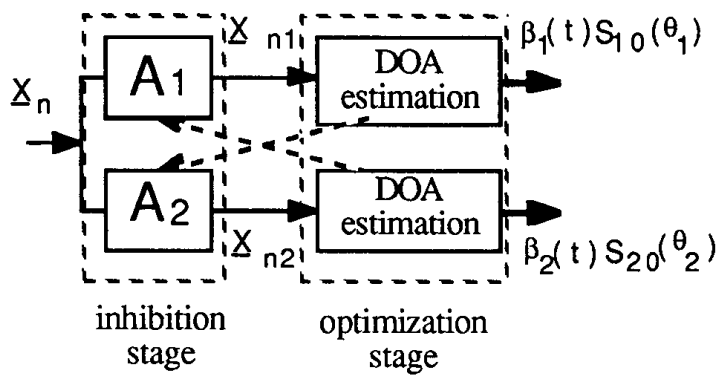

Figure 1. Two stage network for estimation of sources

\section{THE INHIBITION STAGE}

This paper develops an indirect inhibition which consists in the inhibition of a given source by a blocking matrix, represented by $A_{i}$ in figure 1 . Each of the $A_{i}$ matrixes has to cancel one of the two input sources. The direction of this source is obtained by the output of the other system branch.

Note that for the narrowband array problem and given $\Theta_{2}$, the blocking matrix $A_{1}((Q-1) \times Q)$ will be formed as follows, being $Q$ the number of array sensors:

$$
A_{1}=\left(\begin{array}{cccccc}
1 & -\alpha_{1} & 0 & \ldots & 0 \\
0 & 1 & -\alpha_{2} & 0 & \ddots & 0 \\
\vdots & 0 & \checkmark & \checkmark & \checkmark & 0 \\
0 & 0 & 0 & \ldots & 1 & -\alpha_{Q-1}
\end{array}\right)
$$

where:

$$
\alpha_{q}=\exp \left(\frac{j 2 \pi f_{c}}{c}\left(d_{q} \cos \varphi_{q}-d_{q+1} \cos \varphi_{q+1}\right) \sin \theta_{2}\right.
$$

This matrix modifies the original steering vector $S_{1}$ reducing its dimension from number of sensors to number of sensors minus one. The original steering vector can be easily recovered from the modified one $S_{1}$ as it is indicated:

$$
\begin{aligned}
& {\underline{S^{\prime}}}_{1}=\underline{\underline{A}}_{1} \\
& \left(\begin{array}{l}
S_{1}^{\prime}(1) \\
S_{1}^{\prime}(2) \\
\vdots \\
S_{1}^{\prime},(Q-1)
\end{array}\right)=\left(\begin{array}{ccccc}
1 & -\alpha_{1} & 0 & \ldots & 0 \\
0 & 1 & -\alpha_{2} & 0 & \vdots \\
\vdots & \checkmark & \checkmark & & \vdots \\
0 & \ldots & 1 & -\alpha_{Q-1}
\end{array}\right)\left(\begin{array}{l}
S_{1}(0) \\
S_{1}(1) \\
\vdots \\
S_{1}(Q-1)
\end{array}\right) \\
& S_{1}(0)=1 \\
& S_{1}(n)=\frac{S_{1}(n-1)-S_{1}^{\prime}(n)}{\alpha_{n}}
\end{aligned}
$$

In order to gain more insight, the case of a linear equally spaced array is considered. Thus, all the $\alpha_{i}$ s will have the same value:

$$
\alpha=\exp \left(\frac{j 2 \pi f_{c}}{c} d \sin \theta_{2}\right)
$$

where $d$ is the distance between sensors.

This blocking matrix can be represented as:

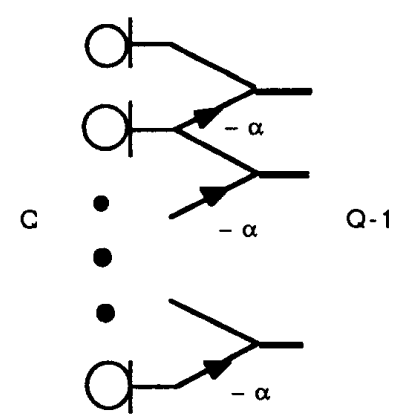

Figure 2. Blocking matrix 


\section{THE OPTIMIZATION STAGE}

The second stage is devoted to find a single variable or waveform from each of the branches of the first stage processor. In our case, only the estimation of the arrival angle of each source is needed.

It is well-know that the signal subspace can be estimated obtaining the NS (number of sources) eigenvectors corresponding to the NS highest eigenvalues of the array correlation matrix. Considering again only two impinging sources, if we manage to cancel one source at the output of the first stage of one of the two branches, this output will have only information about the other source. Then, the eigenvector corresponding to the greatest eigenvalue must be equal to the steering vector of this source. And it will be possible to find the DOA from the maximum of the resulting beamforming:

$$
\left|S_{i}{ }^{H}{ }^{2}{ }_{\max }\right|^{2}
$$

Where each $S_{i}$ is the steering vector of a possible direction of arrival and $e_{\max }$ the greatest eigenvector.

The resulting DOA is used to modify the initial blocking matrix on the other branch.

It is important to notice that this maximization stage works in a coloured noise background which appears at the output of the inhibition stage. The correlation matrix is given by:

$$
\underline{\underline{B}}_{\mathrm{xx}}=\underline{\mathrm{A}} \mathrm{S} \underline{\underline{R}}_{\mathrm{S}} \mathrm{S}^{\mathrm{H}} \underline{\underline{A}}^{\mathrm{H}}+\sigma^{2} \underline{\underline{A}} \underline{\underline{A}}^{\mathrm{H}}
$$

Where $A$ denotes the blocking matrix and $R_{s s}$ is the correlation matrix of the input signals.

In this case the vector we need is:

$$
\underline{\underline{A}} \stackrel{{ }^{H}}{=}{ }^{\mathrm{e}}
$$

Where $e_{1}$ is the generalized eigenvector (GEV) of $R_{\mathbf{x x}}$ corresponding to the largest generalized eigenvalue (GE).

\section{SIMULATION RESULTS}

The presented simulations have been made with a linear equally spaced array of 8 sensors, in which the interelement separation was half wavelength.

The scenario was formed only by two sources with a SNR of $3 \mathrm{~dB}$ each of them. then, the inhibition stage consisted of two blocking matrixes. Each of these matrixes needed a parameter $\left(\theta_{j}\right)$ which had to correspond with the angle of arrival to be cancelled. One of these parameters has been initialized to -30 degrees and the other to 30 . In order to assign the correct values to these parameters a number of iterations had to be done. The following graphics show the final beamforming after only 10 iterations. For each iteration, in the first place, the correlation matrix at the output of each blocking matrix was estimated with 1000 snapshots, after that, the maximum eigenvectors were computed and finally the blocking matrixes were updated.

In figure 3, the resultant beamformer can be seen, when the sources were at 20 and - 10 degrees. In this case the effect of the coloured noise was not important, whether the noise was prewhite or not, the beamformer provides similar performance. The estimated DOAs were 20.99 and -11.08 degrees prewhitening the noise, and $21.17,-11.32$ degrees in coloured noise background.

However when input sources are close together the noise has to be prewhited because with coloured noise the system fails in locating the DOAs. This effect can be seen at figure 4 where the input sources were located at -1 and 6 degrees. On the other hand with the proposed procedure to prewhite the noise, the two directions of arrival could be well estimated: -0.99 and 4.29 degrees (figure 5).

\section{CONCLUSIONS}

A procedure to solve the problem of simultaneous DOA estimation, based on the Kolmogorov's theorem, has been reported.

It is important to remark that although the analysis has been restricted to the case of only two sources, it could be easily extended to any number of them. The design turns the problem from a scenario with NS sources into NS easier problems of single source DOA estimation. The complexity of this method is very low and the results yielded by this approach are quite good.

Some possible improvements to the method are being investigated for the authors. One of them consists in modifying the learning rule applying high order processing in order to guarantee the behaviour of the system even under coherent sources scenarios. For instance, a learning related with the separation between estimated angles of arrival can be defined.

\section{REFERENCES}

[1] Jutten C. "Calcul neuromimetique et traitement du signal: Analyse en composants independents", $\mathrm{Ph}$. Tesis, in french, INPG-USMG Grenoble, 1987.

[2] Common P., Jutten C., Herault J., "Blind separation of sources Part II: Problem statement", 
Signal Processing, vol 24, n 1, pp. 11-21, July 1991.

[3] M. A. Lagunas, A. Pagés, "Multitone tracking with coupled EKFs and high order learning". Proc. ICASSP-92, pp. V153-V156, San Francisco, USA.

[4] M. A. Lagunas, A. Pérez-Neira, "EKF schemes in array processing", NATO-ASI, Acoustic Signal Processing for Ocean Exploration, Madeira, July 26-August 7, 1992.

[5] Kolmogorov A. N., "On the representation of continuous functions of many variables by superposition of continuous functions of one variable and addition". Dokl. Akad Nauk (USSR), in Russian, 114, 952-956, 1957.

[6 Hecht-Nielsen, R., "Neurocomputing", pp. 122124, Addison-Wesley Publishing Company.

[7] Lorentz G., "Approximation of functions", pp. 168-179, Chelsea Publishing Co., New York 1986.

[8] M. A. Lagunas, A. Pérez-Neira, M. Nájar, A. Pagés, "The Kolmogorov Signal Processor", IWANN' 93, Barcelona, Spain, 9-11 June, 1993.

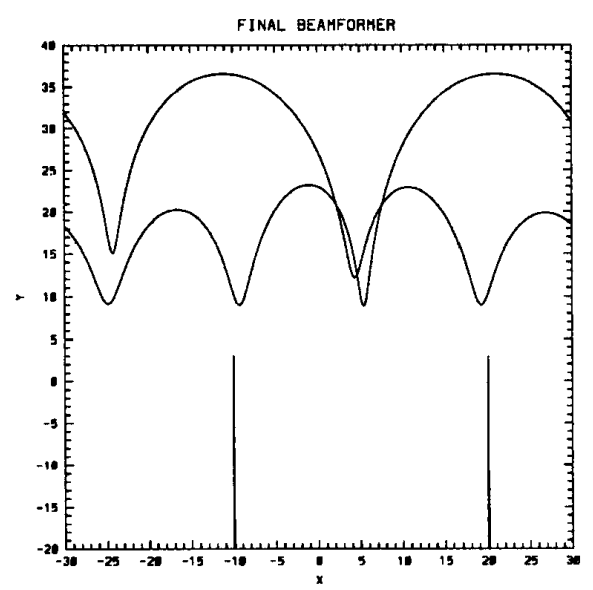

Figure 3.

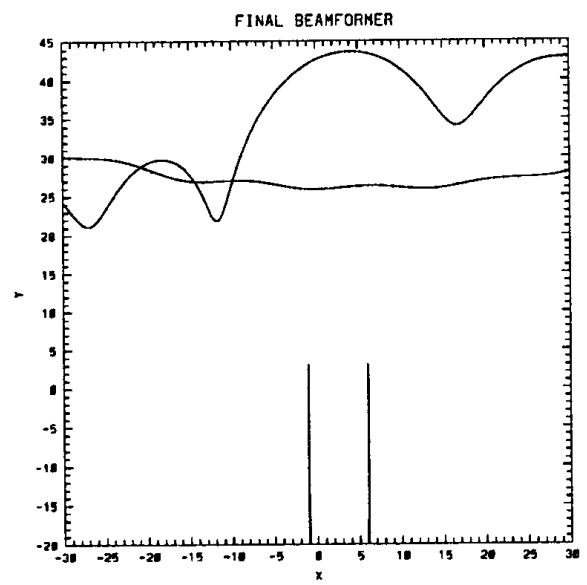

Figure 4.

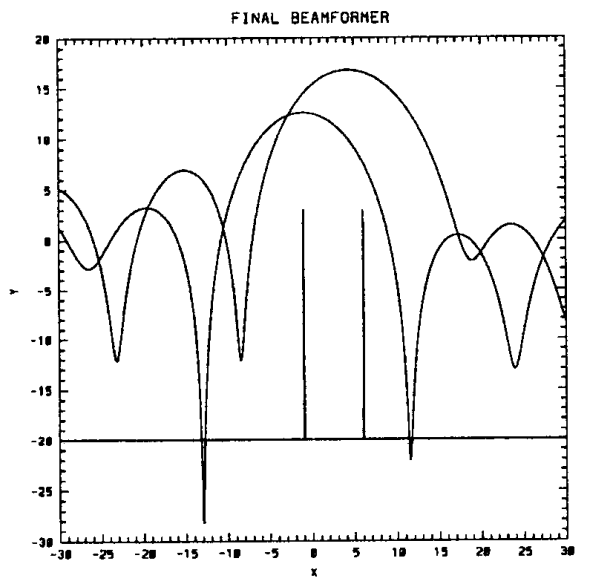

Figure 5 . 